\title{
Mitochondrial deficits in human iPSC-derived neurons from patients with 22q11.2 deletion syndrome and schizophrenia
}

\author{
Jianping Li', Sean K. Ryan², Erik Deboer ${ }^{3}$, Kieona Cook ${ }^{4}$, Shane Fitzgerald ${ }^{5}$, Herbert M. Lachman ${ }^{6}$, \\ Douglas C. Wallace (10), Ethan M. Goldberg $\mathbb{1 0}^{8}$ and Stewart A. Anderson ${ }^{9}$
}

\begin{abstract}
Schizophrenia (SZ) is a highly heterogeneous disorder in both its symptoms and risk factors. One of the most prevalent genetic risk factors for $\mathrm{SZ}$ is the hemizygous microdeletion at chromosome 22q11.2 (22q11DS) that confers a 25-fold increased risk. Six of the genes directly disrupted in 22qDS encode for mitochondrial-localizing proteins. Here, we test the hypothesis that stem cell-derived neurons from subjects with the 22q11DS and SZ have mitochondrial deficits relative to typically developing controls. Human iPSCs from four lines of affected subjects and five lines of controls were differentiated into forebrain-like excitatory neurons. In the patient group, we find significant reductions of ATP levels that appear to be secondary to reduced activity in oxidative phosphorylation complexes I and IV. Protein products of mitochondrial-encoded genes are also reduced. As one of the genes deleted in the 22q11.2 region is MRPL40, a component of the mitochondrial ribosome, we generated a heterozygous mutation of MRPL40 in a healthy control iPSC line. Relative to its isogenic control, this line shows similar deficits in mitochondrial DNA-encoded proteins, ATP level, and complex I and IV activity. These results suggest that in the 22q11DS MRPL40 heterozygosity leads to reduced mitochondria ATP production secondary to altered mitochondrial protein levels. Such defects could have profound effects on neuronal function in vivo.
\end{abstract}

\section{Introduction}

Schizophrenia (SZ) is a heterogeneous disorder generally characterized by adolescent onset of psychosis together with deteriorations of social and cognitive functioning ${ }^{1,2}$. Metabolic compromise, including mitochondrial dysfunction, has long been posited to contribute to the development of symptoms in $\mathrm{SZ}^{3,4}$. Recent studies have further strengthened this notion. For example, gene expression alterations in postmortem neocortical pyramidal neurons suggest disruption of mitochondrial function $^{5}$, and other studies reported mitochondrial

\footnotetext{
Correspondence: Stewart A. Anderson (sande@pennmedicine.upenn.edu) 'Department of Psychiatry, Children's Hospital of Philadelphia, Philadelphia, PA, USA

2Department of Psychiatry, The Children's Hospital of Philadelphia and the University of Pennsylvania Perelman School of Medicine, Philadelphia, PA, USA Full list of author information is available at the end of the article.

*These authors contributed equally: Jianping Li, Sean K. Ryan
}

defects in neural progenitors and cortical interneurons $(\mathrm{cINs})^{6}$ derived from induced pluripotent stem cells (iPSCs) from patients with SZ relative to healthy controls ${ }^{7}$. However, despite the potential importance to the discovery of novel treatments for SZ, evidence for mitochondrial dysfunction in SZ in living, human neurons with a defined genetic alteration has been lacking.

The most prevalent genetic risk factor for SZ is the 22q11.2 deletion syndrome (22q11DS), occurring in about 1:4000 births ${ }^{8}$. Roughly one-quarter of people with the 22q11DS, a 25-fold increase from the general population, develop SZ in a manner that is not grossly distinguishable from non-syndromic SZ ${ }^{9,10}$. Remarkably, in 22q11DS, six of the $\sim 45$ deleted genes encode for proteins that are mitochondrial-localizing, and three others strongly influence mitochondrial function ${ }^{11,12}$. One study has found evidence for mitochondrial dysfunction in serum samples from

\section{(c) The Author(s) 2019}

(c) (i) Open Access This article is licensed under a Creative Commons Attribution 4.0 International License, which permits use, sharing, adaptation, distribution and reproduction c. in any medium or format, as long as you give appropriate credit to the original author(s) and the source, provide a link to the Creative Commons license, and indicate if changes were made. The images or other third party material in this article are included in the article's Creative Commons license, unless indicated otherwise in a credit line to the material. If material is not included in the article's Creative Commons license and your intended use is not permitted by statutory regulation or exceeds the permitted use, you will need to obtain permission directly from the copyright holder. To view a copy of this license, visit http://creativecommons.org/licenses/by/4.0/. 
subjects with the 22q11DS ${ }^{12}$, while another recent study used proteomic analyses in fibroblasts from 22q11DS subjects, together with analyses of 22q model transgenic mice and flies to conclude that mitochondrial SLC25A1/A4 and TXNRD2 influence synaptic function ${ }^{13}$. The importance of Txnrd2 in long-range cortical connectivity and psychosisrelated cognitive deficits was further established recently in transgenic mice ${ }^{14}$. Finally, MRPL4O was identified as a candidate SZ risk gene ${ }^{15}$, and transgenic mice lacking one copy of Mrpl40 show alterations in mitochondrial calcium as well as psychosis-related cognitive deficits ${ }^{16}$. However, to our knowledge, no evidence for mitochondrial dysfunction in living, human neurons from 22q11DS with SZ has been reported.

Here, we have studied mitochondrial function in iPSCderived neurons from patients with 22q11DS + SZ (22qSZ) versus healthy controls. Patient-derived neurons have reduced ATP levels, and reduced activity of complexes I and IV of the electron transport chain (ETC). The levels of multiple mitochondrial-translated proteins are reduced, in contrast to the levels of several nuclearencoded mitochondrial proteins. These findings were replicated in an iPSC line that we edited to be heterozygous for MRPL40. These results suggest that defects in mitochondrial ATP production secondary to reduced levels of mitochondrial-encoded proteins may contribute to neuronal dysfunction in the $22 \mathrm{qSZ}$.

\section{Materials and methods Human iPSCs}

Human iPSCs were contributed by Herbert Lachman (Albert Einstein University), and all of those from patients with 22q11DS also were diagnosed with $\mathrm{SZ}^{17}$. All cell lines had SALSA MLPA KIT P250-A1 DiGeorge (MRC Holland, Amsterdam, Netherlands) testing for the 22q11.2 deletion $^{18}$, and the 22q11.2 deletion lines were confirmed to be hemizygous for the $3 \mathrm{mb}$ deletion located in "A-D" region. In addition, each line was tested monthly and confirmed to be free of mycoplasma. Human iPSCs from four lines of affected subjects and five lines of controls were applied in this study.

\section{Lentiviral vector generation}

Plasmids of VSVG.HIV.SIN.cPPT.CMV.mNgn2.WPRE and VSVG.HIV.SIN. cPPT.CMV.rTTA. WPRE were kindly provided by Dr. Marius Wernig (Stanford University), and packaged into virus by the University of Pennsylvania Viral Vector Core (VSVG.HIV.SIN.cPPT. CMV.mNgn2. WPRE and VSVG.HIV.SIN. cPPT.CMV. rTTA.WPRE.)

\section{Neuronal differentiation of iPSCs}

Induced differentiation of human iPSC lines to neurons (iNrns) was accomplished by an established protocol ${ }^{19}$. In brief, iPSC lines were infected with two lentiviral vectors: TetO-mNgn2-T2A-PuroR and Ubiq-rTTA. On day 1, differentiation was initiated with exposure to doxycycline ( $2 \mu \mathrm{g} / \mathrm{ml}$, Sigma), followed by puromycin $(5 \mu \mathrm{g} / \mathrm{ml}$, Sigma) selection for cells that possessed these two lentiviral vectors $24 \mathrm{~h}$ later. The cells were replated the next day and grown in Neurobasal-A medium (Gibco) with B27 (Life Technologies), glutamax (Life technologies), $5 \mathrm{mM}$ glucose, $10 \mathrm{mM}$ sodium pyruvate, $10 \mathrm{ng} / \mathrm{ml}$ NT-3 (Peprotech), $10 \mathrm{ng} / \mathrm{ml}$ Brain-derived neurotrophic factor (Peprotech), and $2 \mu \mathrm{g} / \mathrm{ml}$ doxycycline. Rat glia, if used, were added on day 5 , and a single administration of $2 \mu \mathrm{M}$ Ara-C (Sigma) was added on day 7. Doxycycline was discontinued on day 10. iNrns were cultured until at least day 21.

\section{Rat glia}

Isolated E17 Sprague Dawley rat cortex was obtained from the UPenn NRU Core. Cells were plated onto uncoated $10 \mathrm{~cm}$ dishes at 1.5 million cells $/ \mathrm{ml}$. Cells were grown in NM-15 media (Eagle's MEM with Earle's salts and $2 \mathrm{mM}$ L-glutamine, $15 \%$ heat-inactivated fetal bovine serum, $6 \mathrm{mg} / \mathrm{ml}$ glucose, $0.5 \mathrm{U} / \mathrm{ml}$ penicillin, and $0.5 \mu \mathrm{g} / \mathrm{ml}$ streptomycin). Medium exchanges occurred every 5 days, and rat glia grew for at least 2 weeks. Prior to glial harvest, the glia were shaken at $37^{\circ} \mathrm{C}$ for $3 \mathrm{~h}$ at $230 \mathrm{rpm}$ to remove microglia. The glia were then dissociated with accutase (Life Technologies), spun down for $5 \mathrm{~min}$ at $1000 \mathrm{rpm}$, resuspended in neurobasal/B27 media, and plated onto the neurons at $100 \mathrm{k}$ cells/well of a 24 well plate.

\section{Western blot}

Cells were washed with ice-cold PBS twice. Ice-cold RIPA buffer and protease inhibitor cocktail were added next, and cells were scraped from the plate and put into a 1.5-ml Eppendorf tube. Cells were placed on ice for $30 \mathrm{~min}$, then centrifuged at $14,000 \mathrm{rpm}$ for $15 \mathrm{~min}$ at $4{ }^{\circ} \mathrm{C}$. Supernatant was collected and frozen at $-80^{\circ} \mathrm{C}$ until needed. Protein concentration was quantified with the Pierce BCA kit and a NanoDrop2000 (Thermo scientific) to measure. Samples were loaded on a $4-12 \%$ bis-tris gel with LDS Sample Buffer (Life technologies) and Sample Reducing Agent (Life technologies). The SDS-PAGE was run with MOPS buffer (Life Technologies), protein was transferred from gel to nitrocellulose membrane, which was then blocked for $1 \mathrm{~h}$ at room temperature using 5\% BSA. Li-Cor/Odyssey and Image J are used for data collection and analysis. We used the following primary antibodies: anti-MRPL40 (1:500; Novus), anti-VDAC (1:1000; Neuromab), anti-cytochrome b (1:200; Santa Cruz Biotechnology), anti-MT-ND1 (1:500; Abcam), antiOXPHOS cocktail (1:250; abcam), and $\beta$-actin (1:10,000; Cell Signaling Technology). The following Licor 
secondary antibodies were used all at 1:10,000: IRDye 680LT Goat anti-Mouse, IRDye 680RD Donkey antiRabbit, IRDye 800CW Donkey anti-Rabbit, IRDye 800CW Donkey anti-Goat, IRDye 800CW Donkey anti-Mouse.

RNA extraction, reverse transcription, and quantitative PCR Total RNA was extracted using Trizol (Ambion) and the concentration was measured using nanoDrop2000 spectrophotometer. cDNA was generated using transcript IV VILO Master Mix (Thermo Fisher). RNA abundance was measured by Quantitative PCR using TaqMan Gene Expression Master Mix (Applied Biosystems) for primer pairs of ACTIN, MRPL40, VDAC1, and COX1 purchased from Thermo Fisher Scientific, and SYBR Green PCR Mix (Applied Biosystems) for primer pairs of cytochrome $b$ and mt-ND1. Primer sequences: ACTIN (Assay ID: Hs01060665_g1), MRPL40 (Assay ID: Hs00186843_m1), VDAC1 (Assay ID: Hs01019083_m1), COX1 (Assay ID: Hs02596864_g1); cytochrome b: forward primer: 5'-AGTCCCACCCTCACACGATTCTTT3', reverse primer: 5'-AGTAAGCCGAGGGCGTCTTTGATT-3'; mt-ND1: forward primer: 5'-ATGGCCAACCTCCTACTCCTCATT-3', reverse primer: 5'TTATGGCGTCAGCGAAGGGTTG TA-3'.

\section{Synapse counting}

Induced neurons grown to day 21 with rat glia were fixed in 4\% PFA, stained with DAPI (1:2000; Invitrogen), VGLUT1 (1:1000; Sigma), PSD95 (1:500; Neuromab), and MAP2 (1:500; Abcam). The respective secondary antibodies were Donkey Anti-Rabbit Alexa 488 (1:500; Thermo Fisher Scientific), Goat anti-Mouse Alexa 568 (1:500; Thermofisher Scientific), and Goat anti-Chicken 680 (1:500; Thermo Fisher Scientific). Then, 40× images were collected using a Leica DMI8 confocal microscope. At least three images were collected from three control and three schizophrenic lines of cells. Each neuron imaged had no less than two major processes. After collection, images were deconvolved using Hyguens Essential software. Next, images were transferred to Imaris software, where they were then cropped and surfaced by MAP2 staining. PSD95 puncta about $0.5 \mu \mathrm{m}$ in diameter were identified within the MAP2-positive dendrites, then VGLUT1 puncta of $0.5 \mu \mathrm{m}$ localized within $1 \mu \mathrm{m}$ of the PSD-95 were identified. These co-localized puncta within $40 \mu \mathrm{m}$ of the cell soma were quantified as synapses. Cells were counted to normalize for culture density by selecting random areas and counting number of DAPI+MAP2+ cells per unit area.

\section{Preparation of cell lysate for mitochondrial OXPHOS activity}

The preparation of cell lysate was conducted as published $^{20}$. Briefly, induced neurons at day 21 were washed with cold PBS, then suspended and centrifuged at $1000 \mathrm{rpm}$ for $5 \mathrm{~min}$. Cell pellets were stored at $-80^{\circ} \mathrm{C}$ until measurement of OXPHOS activity.

\section{Measurement of mitochondria OXPHOS activity and ATP levels}

Biochemical methods were used as described ${ }^{21}$. Briefly, cell pellets were thawed, then flash frozen in liquid nitrogen three times. Cell lysates were placed into a cuvette with reaction buffer and recorded at the relevant wavelength for each complex assay. Potassium buffer was used in all enzyme activity assays and contains $50 \mathrm{mM}$ $\mathrm{KCl}, 10 \mathrm{mM}$ Tris- $\mathrm{HCl}$, and $1 \mathrm{mM}$ EDTA with $\mathrm{pH} 7.4$. Reagents for detection of complex I enzyme activity (wavelength $340 \mathrm{~nm}$ ) include $5 \mathrm{mM} \mathrm{MgCl} 2,2 \mathrm{mM} \mathrm{KCN}$, $0.13 \mathrm{mM}$ fresh $\mathrm{NADH}, 65 \mu \mathrm{M} \mathrm{CoQ}, 2 \mu \mathrm{g} / \mathrm{ml}$ antimycin, and $2 \mu \mathrm{g} / \mathrm{ml}$ rotenone. Reagents for complex II activity (wavelength $600 \mathrm{~nm}$ ) consist of $5 \mathrm{mM} \mathrm{MgCl}_{2}, 20 \mathrm{mM}$ succinate, $2 \mathrm{mM} \mathrm{KCN}, 65 \mu \mathrm{M} \mathrm{CoQ} 2,2 \mu \mathrm{g} / \mathrm{ml}$ antimycin, $2 \mu \mathrm{g} / \mathrm{ml}$ rotenone, and $50 \mu \mathrm{M}$ dichlorophenolindophenol. Reagents used for complex III activity (wavelength $550 \mathrm{~nm}$ ) include $5 \mathrm{mM} \mathrm{MgCl} 2,2 \mathrm{mM} \mathrm{KCN}, 15 \mathrm{mM}$ fresh cytochrome c, $65 \mu \mathrm{M} \mathrm{CoQ}_{2}, 0.6 \mathrm{mM}$ dodecyl- $\beta$-d-maltoside, and $2 \mu \mathrm{g} / \mathrm{ml}$ rotenone. The reagent used for complex IV activity (wavelength $550 \mathrm{~nm}$ ) is $15 \mu \mathrm{M}$ cytochrome c. Complex V (ATP synthase) enzyme activity Microplate Assay Kit (Abcam, ab109714) was applied following manufacturer's instructions. All chemicals for enzyme analyses were purchased from Sigma-Aldrich.

ATP levels were quantified on a Luminescence plate reader using an ATP Bioluminescence Assay Kit (Abcam) following the manufacturer's instructions. Briefly, cells were lysed by detergent provided in the kit, followed by addition of reconstituted substrate solution and measurement of luminescence. All procedures were performed in the dark.

\section{Generation of MRPL40 heterozygous line}

The MRPL4O heterozygous line was generated as described $^{22}$. Guide RNAs were designed using http://crispr.mit.edu to locate to exon 2 of MRPL40. Guide RNAs were ligated into Fast BbsI digested pSpCas 9 (BB)-GFP vectors (Addgene, plasmid ID: 48138). The cloned gRNA-Cas9n vectors were introduced into human iPSCs by electroporation under program B16. Human stem cell Nucleofector Kit1 (Lonza) was used. SURVEYOR assays (Transgenomic, cat. no. 706025), DNA sequencing, and western blot were used to validate the MRPL40 heterozygous mutation. Sequence of gRNAs: gRNA-Top: 5'-CACCGAAGACAACAATGACGCTCG C-3'; gRNA-Bottom: 5'AAACGCGAGC GTC ATTGT TGTCTTC-3'. MRPL40 primers: Forward primer: $5^{\prime}-\mathrm{C}$ CTTCCACGTTGACCTTGCT-3'; Reverse primer: 5'-CC TTCCACGTTGACCTTGCT-3'. 


\section{Mitochondrial DNA copy number}

All reactions were performed in fast optical 96-well reaction plates with barcodes (Applied Biosystems) on SDS7900HT system (CHOP NAPCore). Each sample was analyzed in triplicate. The reaction solution contains $2 \mu \mathrm{l}$ of DNA template $(3 \mathrm{ng} / \mu \mathrm{l}), 2 \mu \mathrm{l}$ of primers $(5 \mu \mathrm{m}), 12.5 \mu \mathrm{l}$ of SYBR Green PCR Master Mix (Applied biosystems), and $8.5 \mu \mathrm{l}$ of $\mathrm{H}_{2} \mathrm{O}$. The procedure of amplification program was as follows: $10 \mathrm{~min}$ at $95^{\circ} \mathrm{C}, 40$ cycles of $15 \mathrm{~s}$ at $95^{\circ} \mathrm{C}$, and $60 \mathrm{~s}$ at $60^{\circ} \mathrm{C}$ and melting curve. Relative mtDNA copy number (mtDNA amount/nDNA amount) was calculated by a comparative $\mathrm{Ct}$ method, using the following equation: $\mathrm{mtDNA} / \mathrm{nDNA}=2^{-\Delta \mathrm{Ct}}$. Nuclear primers includes LPL-F: 5'-CGAGTCGTCTTTCTCCT GATGAT-3', LPL-R: 5'-TTCTGGATTCCAATGCTTC GA-3', B2-microglobulin-F: 5'-TGCTGTCTCCATGTTT GATGTATCT-3' and B2-microglobulin-R: 5'-TCTCT GCTCCCCACCTCTAAGT-3'. Mitochondria primers contain tRNA Leu(UUR)-F: 5'-CACCCAAGAACAGGG TTTGT-3', tRNA Leu(UUR)-R: 5'-TGGCCATGGG TATGTTGTTA-3'; ND1-F: 5'-CC CTAAAACCCGC CACATCT-3', ND1-R: 5'-GCGATGGTGAGAGCTA AGGT-3'; ND4-F: 5'-CCATTCTCCTCCTATCCCT CAAC-3', ND4-R: 5'-CCATTCTCCTCCTATCCCT CAC-3'; cytochrome b-F: 5'-CACGATTCTTTACCTTT CACTTCA TC-3', and cytochrome b-R: $5^{\prime}$-TGATCCCG TTTCGTGCAAG-3'.

\section{Electrophysiology}

Whole-cell recordings were obtained from neurons with patch pipettes pulled from borosilicate glass (outer diameter, $1.5 \mathrm{~mm}$; inner diameter, $0.86 \mathrm{~mm}$ ) pulled on a horizontal puller (P-97, Sutter Instruments) and filled with intracellular solution that contained, in $\mathrm{mM}$ : K-gluconate, 130; $\mathrm{KCl}$, 6.3; EGTA, 0.5; $\mathrm{MgCl}_{2}$, 1.0; HEPES, 10; Mg-ATP, 4.0; Na-GTP, 0.3. $\mathrm{pH}$ was adjusted to 7.30 with $\mathrm{KOH}$; osmolality was adjusted to $285 \mathrm{mOsm}$ with $30 \%$ sucrose. When filled with internal solution, pipettes had a resistance of 5-7 M $\Omega$. Calculated chloride equilibrium potential was $-73 \mathrm{mV}$. Unless otherwise specified, chemicals were purchased from Sigma-Aldrich (St. Louis, MO, USA).

Recordings were performed with a MultiClamp 700B amplifier (Molecular Devices, Sunnyvale, CA) using pCLAMP 10 software. Pipette capacitance and series resistance compensation (bridge balance) were applied throughout current-clamp experiments, with minor bridge balance re-adjustments allowed as required. Signals were low-pass filtered at $10 \mathrm{kHz}$ and sampled at $20-50 \mathrm{kHz}$ and digitized using a Digi data 1550A 16-bit D/A converter (Molecular Devices). Reported values for membrane potential $\left(V_{\mathrm{m}}\right)$ and action potential threshold are not corrected for the liquid junction potential.

Spontaneous resting membrane potential $\left(V_{\mathrm{m}}\right)$ was determined $2 \mathrm{~min}$ after break-in as the average membrane potential during a 1-s sweep with no current injection. For cells that were spontaneously active, this was measured in the inter-spike interval. Input resistance $\left(R_{\mathrm{m}}\right)$ was calculated as the slope of the linear fit to the plot of the $V-I$ relation derived from small subthreshold current steps at/ around resting membrane potential. Action potential threshold was calculated as the voltage at which the first derivative $(\mathrm{d} V / \mathrm{dt})$ of the AP waveform reached $10 \mathrm{mV} / \mathrm{ms}$. Action potential half-width (AP $1 / 2$-width) is defined as the width of the AP (in ms) at half-maximal amplitude, calculated using AP threshold and the peak of the AP. Action potential after-hyperpolarization (AHP) amplitude is calculated as the depth of the after discharge potential (in $\mathrm{mV}$ ) relative to AP threshold.

Maximal steady-state firing frequency is the maximal mean firing frequency in response to a current injection at which there are no AP failures, with spikes defined as having a clear AP threshold as per above, amplitude of $40 \mathrm{mV}$ or higher, and overshooting $-10 \mathrm{mV}$. Maximal instantaneous firing frequency is the inverse of the smallest inter-spike interval at maximal current step injection.

Cutoff frequency $(\mathrm{Hz})$ is defined as the frequency after which a cell can no longer produce action potentials (i.e., there are action potential failures) in response to repetitive stimulation for $10 \mathrm{~s}$.

Experimental design: Data from 7-10 cells per line were included in the analysis.

\section{Statistical analysis}

Experimental " $N$ " refers to the number of iPSCs lines from different individuals of each group (control $=5$; $22 \mathrm{qSZ}=4$ ) used in each experiment.

Data were presented as mean \pm standard error of the mean (SEM). Unpaired two-tailed Student's $t$-test and Chi-square were used for data analysis. $p<0.05$ was considered a significant difference. Data were analyzed and graphed with GraphPad Prism version 8.

\section{Results}

Human iPSC-derived neurons (iNrns) from subjects with 22q11DS and SZ have reduced ATP levels and ETC activity

To investigate the hypothesis that the pathobiology of SZ in the context of 22q11DS could include mitochondrial dysfunction in neurons, we studied five iPSC lines from healthy controls and four lines from subjects with 22q11DS and SZ (22qSZ) that have been published previously ${ }^{17}$ (Fig. 1a). These lines were differentiated via the induced expression of neurogenin 2 (Fig. 1b), a well-established protocol that rapidly and reproducibly generates a relatively homogenous population of excitatory projection neuronlike cells (Fig. 1c, d and see also Fig. S1) ${ }^{19}$.

We first examined ATP production, finding a roughly $50 \%$ reduction in the $22 \mathrm{qSZ}$ neurons (Fig. $2 \mathrm{a}$ ). To explore 


\begin{tabular}{lcl}
\hline \multicolumn{3}{c}{ Human iPSC line } \\
\hline Line & Age & \multicolumn{1}{c}{ Sex } \\
\hline HC1 & 28 & Female \\
HC2 & 28 & Female \\
HC3 & 31 & Male \\
HC4 & 32 & Female \\
HC5 & 34 & Male \\
22 qSZ1 & 25 & Male \\
22 qSZ2 & 28 & Female \\
22 qSZ3 & 28 & Female \\
22qSZ4 & 32 & Male \\
\hline
\end{tabular}

C

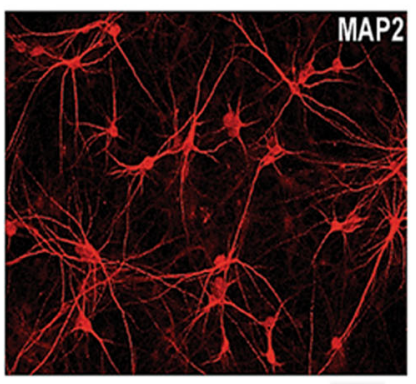

b Lentiviral constructs
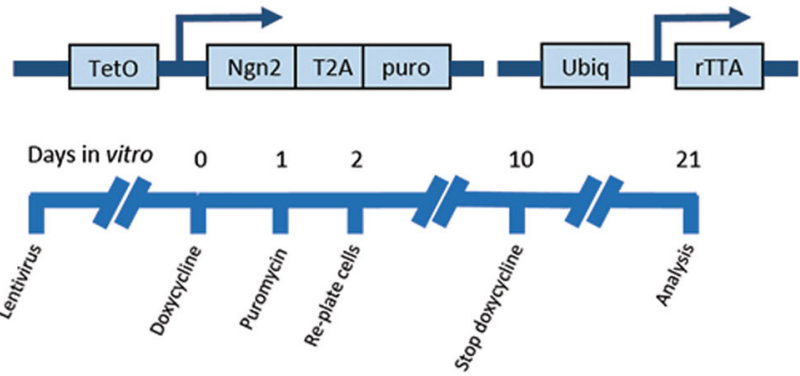

d

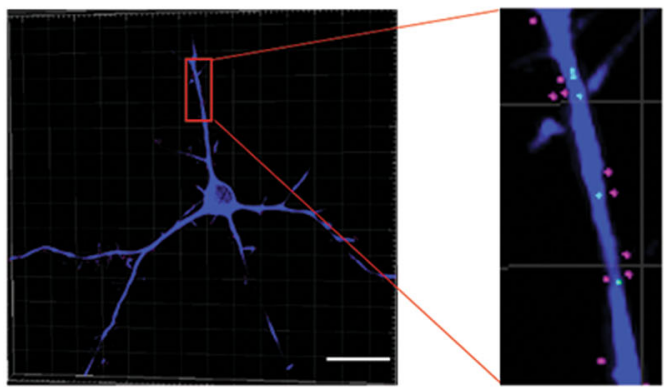

Fig. 1 iPSC lines and induced neuronal differentiation protocol used in this study. a Table showing subject characteristics. $\mathbf{b}$ Lentiviral constructs and timeline used to induce neuronal differentiation. Doxycycline is discontinued at 10 days in vitro (DIV). c Epifluorescence image of a DIV21 culture, from a 22qSZ line, with fluorescence immunodetection of the neuronal dendritic marker MAP2. $\mathbf{d}$ Confocal image processed by deconvolution and Imaris software showing apposition of MAP2 (blue) with the excitatory presynaptic protein vGLUT (green), and the excitatory postsynaptic protein PSD95 (red). Scale bars: $50 \mu \mathrm{m}$ in c, $30 \mu \mathrm{m}$ in $\mathbf{d}$.

potential causes of this decrease, we next investigated enzymatic activity in the ETC. Both complexes I and IV exhibited significantly reduced activity in 22qSZ neurons (Fig. 2b, c); however, complexes II, III, and V (ATP synthase) enzyme activity was unchanged (Fig. 2d, e and Fig. S2).

While all 13 of the mitochondrial DNA-encoded proteins function in the ETC, they are not evenly distributed $^{23}$. The two ETC complexes with significant reductions of activity in the 22qSZ group, complexes I and IV, have seven and three mitochondrial-encoded proteins, respectively. In contrast, complexes II, III, and V have zero, one, and two of these proteins, respectively. The correlation of complex activity reductions to their number of mitochondrial-encoded proteins raises the possibility that an abnormality in mitochondrial translation could be influencing these phenotypes ${ }^{24}$. That possibility was already implicated by the fact that one of the genes deleted in the 22q11DS, MPRL40, encodes for a subunit of the mitochondrial ribosome ${ }^{25}$.

\section{iNrns from subjects with 22qSZ have reduced levels of mitochondrial-encoded proteins}

Since MRPL40 (mitochondrial ribosomal protein L40) assists in protein translation of mitochondria encoded genes, we examined mRNA and protein levels encoded by nuclear and mitochondrial genes that generate mitochondrial proteins. As expected, MRPL40 was reduced at the protein (Fig. 3a) and at the mRNA (Fig. 3e) levels ${ }^{17}$. Importantly, neither protein (Fig. 3a) nor mRNA (Fig. 3e) levels of the nuclear encoded voltage-dependent anion channel (VDAC) were altered in neurons from the 22qSZ group. The nuclear encoded complex II protein SDHA was also unaltered (Fig. 3b), as was the mtDNA copy number (Fig. 3f). Together, these results suggest that there are no gross alterations in mitochondrial mass or DNA in 22qSZ group relative to controls.

Remarkably, mitochondrial DNA-encoded protein levels, including those for MT-ND1 (complex I), cytochrome b (complex III), and COX1 (complex IV) were significantly reduced in neurons from the 22qSZ lines (Fig. 3c, d). However, the mRNAs for these proteins were unaltered (Fig. 3e). Taken together, these results suggest that the reduction of ATP in neurons from the 22qSZderived lines is secondary to reduced levels of mitochondrial-encoded proteins.

\section{Heterozygosity for a truncating mutation in MRPL40 reduces} mtDNA-encoded protein expression and neuronal ATP

To test whether haploinsufficiency for MRPL40 alone is sufficient to compromise ATP levels and mitochondrial 

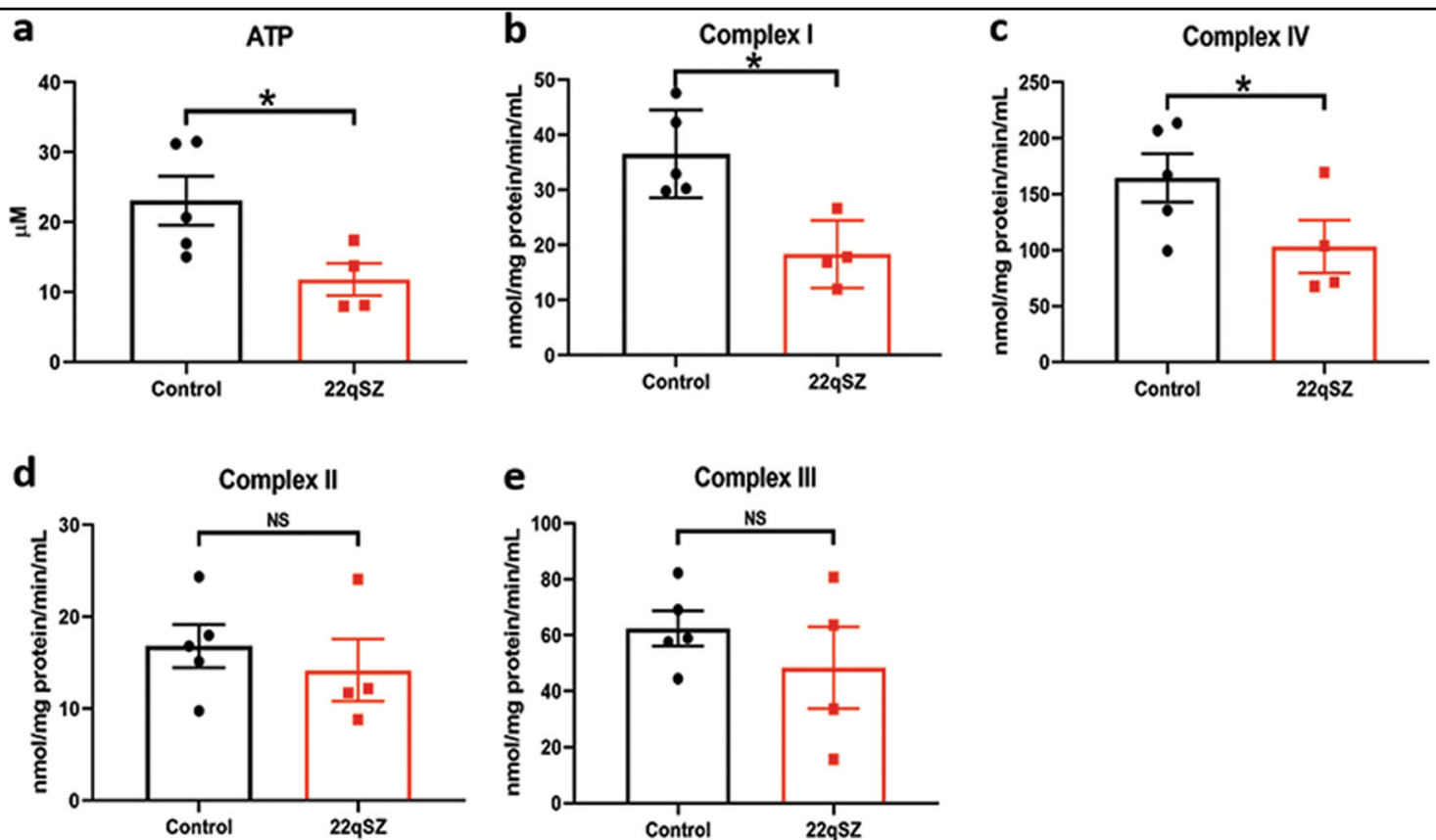

Fig. 2 iNrns from subjects with 22qSZ have reduced ATP levels, and reduced ETC complexes I and IV activity. a ATP level measured by luminescence detection assay at differentiation day 21. Relative to controls, the 22q11DS group had a nearly 50\% reduction of ATP level. b-e Mitochondrial complex I (NADH-ubiquinone oxidoreductase) (b); complex IV (cytochrome c oxidase) (c); complex II (succinate-ubiquinone oxidoreductase) (d); and complex III (ubiquinone-cytochrome c oxidoreductase) (e) were measured in day21 iPSC-derived neurons. Relative to controls, the 22qSZ group had significant reduction of complexes I and IV enzyme activity, but not of complex II or III. $N=5$ for control, $N=4$ for 22qSZ; * indicates $p<0.05$, NS-not significant.

protein translation in human neurons, a loss of function mutation was introduced into one allele of MRPL40 in an iPSC line from a healthy control (Fig. S3). iNrns generated from this line have decreased MRPL40 protein (Fig. 4a) as well as mRNA (Fig. 4c). As found above with the 22qSZ iPSC-derived neurons compared to the healthy control group (Fig. 3), neurons from the $M R P L 40^{+/ m u t}$ line showed no change in the nuclear encoded mitochondrial proteins VDAC or SDHA relative to its isogenic control (Fig. 4a). However, MT-ND1, cytochrome b, and COX1 were again significantly reduced at the protein level (Fig. 4b), but not at the RNA level (Fig. 4c).

These results suggest that reduction of MRPL40 results in reduced translation of mitochondrial proteins. To determine whether the haploinsufficiency mutation for MRPL4O is also sufficient to alter mitochondrial function, ATP levels and ETC complex activities were assessed. Similar to findings from the 22qSZ iNrns, there was a roughly $30 \%$ decrease in ATP in iNrns from the $M R P L 4 O^{+/ m u t}$ line (Fig. 5a). Complex I and IV activities were also significantly reduced (Fig. 5b, c), but not complexes II and III (Fig. 5d, e). Taken together, these results suggest that MRPL4O haploinsufficiency is likely to be an important contributor to the mitochondrial phenotypes identified in iNrns from the 22qSZ group and that the reduction of ATP in iNrns from the
22qSZ-derived lines is secondary to reduced mitochondrial protein translation.

\section{Neurons from subjects with 22qSZ show different cutoff frequency and similar synapse density as controls}

The presence of reduced ATP levels in neurons from $22 \mathrm{qSZ}$ patients raises the question of whether these neurons have functional deficits. To test this possibility, cells were plated onto glass coverslips. Rat astrocytes, which greatly increase synaptogenesis in these cultures, were added at day 5 . The media was changed to Brainphys at day 21 , and then whole cell patch-clamp recordings were performed after 5 weeks in vitro (Fig. S1a). Multiple parameters that could be indicative of neuronal maturational state were unchanged between the 22qSZ and controls, including resting membrane potential $(\mathrm{Rm})$, input resistance, action potential half-width and max steady-state firing frequency (Table S1). On the other hand, measures of parameters thought to test bioenergetics, such as cutoff frequency, show significant difference in $22 \mathrm{qSZ}$ versus controls. In addition, synapse density, measured by confocal microscopy of immunofluorescent-labeled synapses along MAP2-labeled dendrites, was not significantly different between the groups (Fig. S1c). In sum, while the 22qSZ neurons have less tendency to respond to break through of the patch- 
a
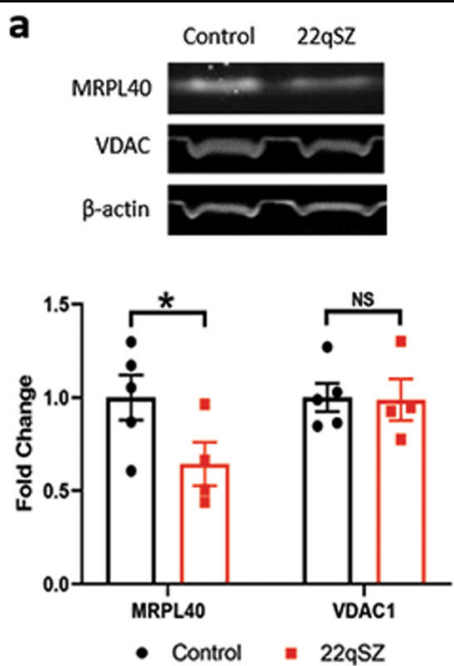

d
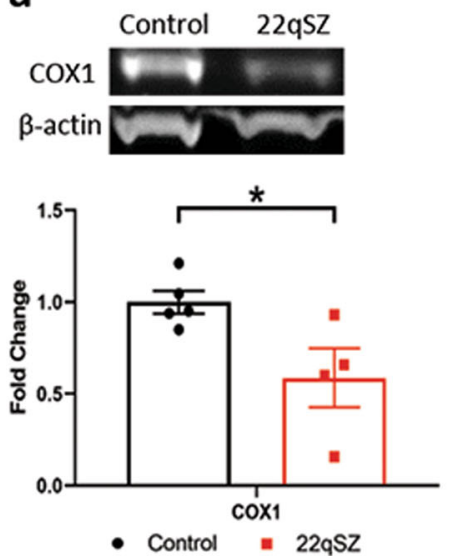
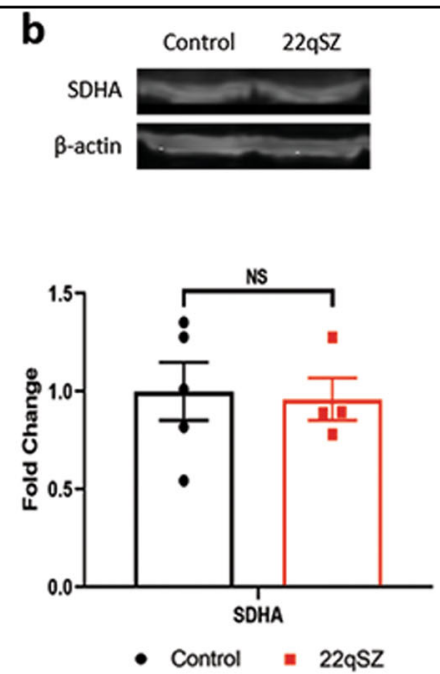

e

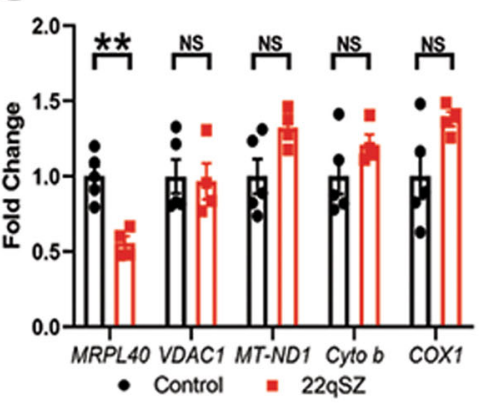

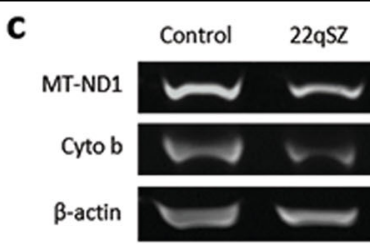

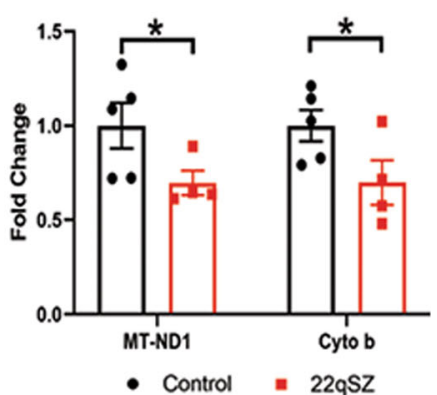

$\mathbf{f}$

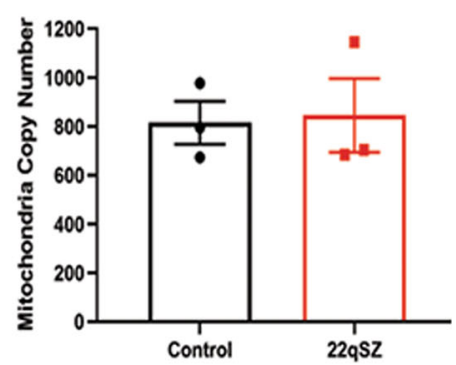

Fig. 3 Reductions in mitochondrial-DNA encoded protein in 22qSZ. a-d Protein levels, by western blot and relative to $\beta$-actin, of MRPL40, VDAC, SDHA, MT-ND1, cytochrome b, and COX1 in iPSC-derived neurons. While nuclear-encoded VDAC and SDHA were unchanged, the mitochondriaencoded proteins MT-ND1, cytochrome $b$, and COX1 were significantly reduced. e Quantitative PCR of MRPL40, VDAC, MT-ND1, cytochrome b, and COX1 shows decreased expression of MRPL40 but no change of VDAC, MT-ND1, cytochrome $b$, and COX1 in $22 q S Z$ relative to control. $\mathbf{f}$ No difference of mitochondrial DNA copy number between 22qSZ and control in iPSC-derived neurons at differentiation day 21. $N=5$ for control, $N=4$ for $22 q S Z$; * indicates $p<0.05$. NS-not significant.

pipette with a spike train, by multiple intrinsic electrophysiological measures as well as synapse density analysis, the 22qSZ iNrns had well-established neuronal characteristics and were not distinguishable from controls at about day 60 .

\section{Discussion}

SZ primarily evolves from the interacting influences of multiple risk-alleles whose effects on brain development can also be influenced by both the prenatal and postnatal environment ${ }^{26,27}$. One of the strongest genetic risk factors for $\mathrm{SZ}$ is the hemizygous microdeletion at chromosome 22 q11.2 that imparts a $25 \%$ risk, or about 25 times that of the general population ${ }^{28}$. Since nine of the roughly 45 genes deleted in 22q11DS generate proteins affect mitochondrial function ${ }^{11,12}$, we examined this function in iPSC-derived neurons. We found that ATP levels were reduced in iPSC-derived neurons from patients with $22 \mathrm{qSZ}$, a phenotype that appears to be caused primarily by reduced activity of complexes I and IV. Interestingly, neurons from the 22qSZ lines also had reduced levels of several mitochondrial-encoded proteins, but not of nuclear encoded proteins. Since complexes I and IV have the highest number of mitochondrial-encoded proteins, these findings raised the possibility that the 22q11DS is associated with reduced neuronal protein synthesis, an idea supported by the presence of MRPL4O in the deleted region. We thus edited a control iPSC line to be hemizygous for MRPL40. Relative to its isogenic control, and like the 22qSZ lines relative to their controls, the 


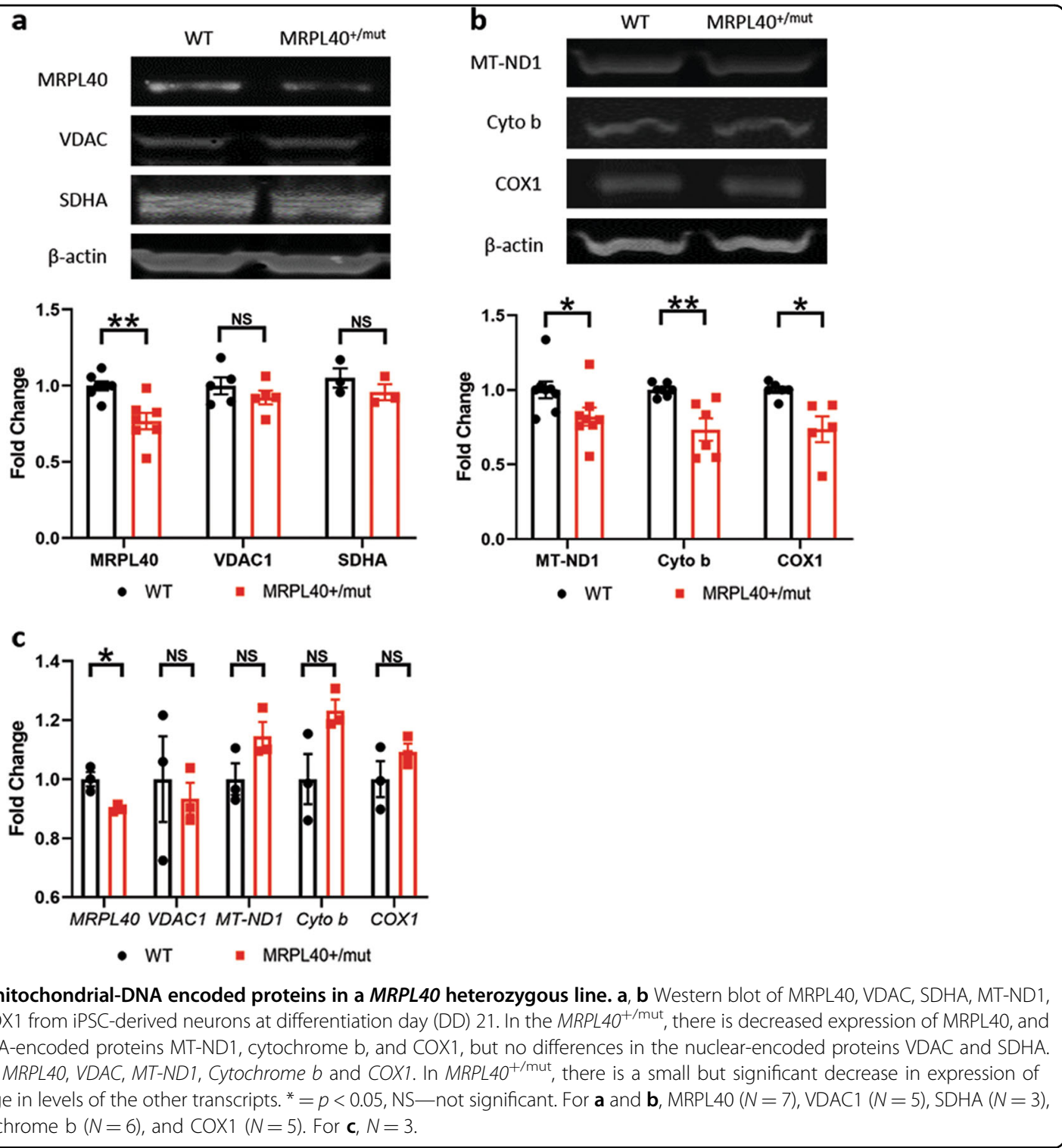

MRPL40 hemizygous neurons have normal levels of mitochondria proteins encoded by nuclear genes, but decreased levels of proteins encoded by the mitochondrial DNA, decreases in ETC complexes I and IV activities, and decreased ATP level. We conclude that hemizygosity at $22 \mathrm{q} 11.2$ is associated with decreased neuronal ATP levels, and that this phenotype is most likely related to decreased mitochondrial protein synthesis. Unfortunately, our attempts to quantify mitochondrial protein synthesis in mature iNrns have been unsuccessful. We believe this is the first demonstration of a SZ-related mutation resulting in a mitochondrial deficit in human neurons.

A key aspect of any study involving the use of human iPSCs to study disease is the cell type chosen. Here, human iPSC lines were differentiated via the induced expression of neurogenin 2, which rapidly generates a relatively homogenous population of excitatory projection neuron-like cells (iNrns) ${ }^{19}$. An advantage of this approach is that reasonably mature neurons can be generated in a matter of weeks, with high degree of consistency across lines. A disadvantage of this approach for the current study is that neurogenin 2 expression is induced by doxycycline, an inhibitor of bacterial mitochondrial protein synthesis that can also affect mammalian mitochondria ${ }^{29}$. Of note, mitochondrial assays were run 11 days following stoppage of the doxycycline in the neuronal induction protocol, but the possibility remains that doxycycline, or even the puromycin exposure for 1 day at the start of the protocol, could be unmasking a vulnerability in the 22qDS and the MRPL40 hemizygous iNrns. 

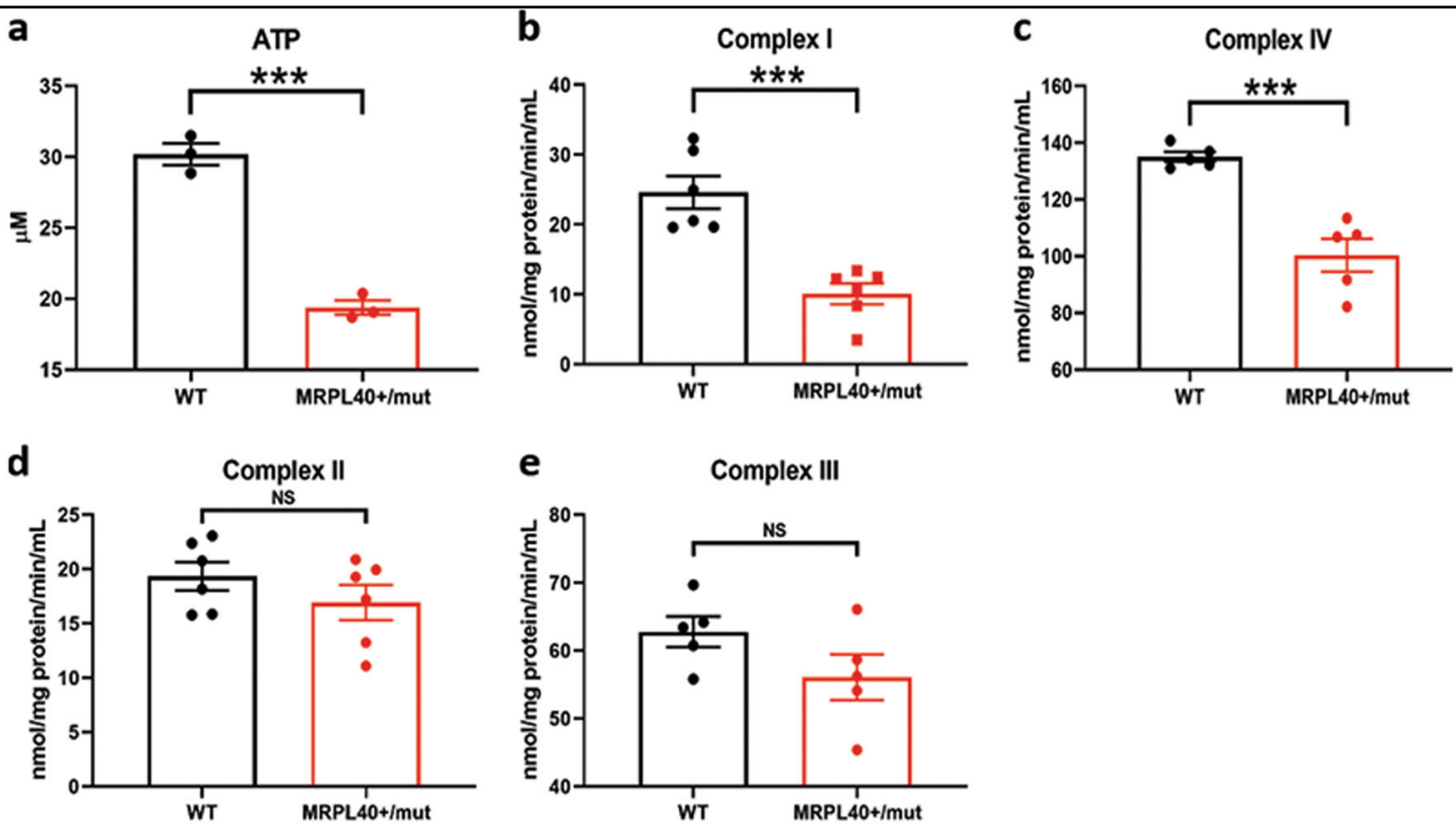

Fig. 5 Neurons derived from MRPL40 $0^{+/ m u t}$ cell show decreased OXPHOS complex I and IV activity and reduced ATP level. a Bar graph shows reduced ATP level in the MRPL4O $40^{+/ m u t}$ neurons $(N=3)$. $\mathbf{b}$-e In $M R P L 40^{+/ \text {mut }}$ neurons, there is also reduced activity in mitochondrial complex I $(\mathbf{b}, N=$ 6) and complex IV $(\mathbf{c}, N=5)$, while complex II $(\mathbf{d}, N=6)$ and complex III $(\mathbf{e}, \mathbf{N}=5)$ activity was not affected. * Indicates $p<0.05$, NS-not significant.

Since mitochondrial gene and protein expression, as well as functional properties, are likely to differ across cell types, the relative uniformity provided by the Ngn2 protocol is another major advantage. In fact, while the lack of neuronal phenotypes such as input synaptogenesis and electrophysiological measures is disappointing, since such phenotypes could secondarily influence mitochondrial function, the high similarities in properties between iNrns from the $22 \mathrm{qSZ}$ and control groups strengthens our confidence that the mitochondria phenotypes themselves are not epiphenomena. That we replicated these phenotypes in the hemizygous MRPL4O line relative to its isogenic control, further bolsters this contention. In future studies, it will be important to assess mitochondrial phenotypes in longer duration culture systems, transplantations, and well as with electrophysiological conditions such as limited ATP and EGTA in the recording solution that might unmask relevant phenotypes. It would also be interesting to study haploinsufficiency for MRPL4O affects calcium signaling, as studied in mouse hippocampal neurons ${ }^{16}$. In addition, other cell types that can show SZ-related phenotypes should be tested, including astrocytes, microglia, and cINs. The "fastspiking" cIN has more mitochondria in its pre-synaptic terminals than other cortical neurons ${ }^{30}$, and has otherwise been associated with high energy requirements, susceptibility to oxidative stress, and $\mathrm{SZ}^{31,32}$. However, to date the fast-spiking subclass of cINs has not been definitively generated, in a highly enriched preparation, from human stem cells.
So what bearing might these findings have to understanding SZ? Of course, we cannot conclude that our findings relate to the SZ-related symptoms of the $22 q 11.2$ subjects in our study. Important results would be obtained from future studies involving the comparison of iPSCs from 22q11DS subjects with versus without SZ. It also bears mention that MRPL4O haploinsufficiency alone is unlikely to account for all mitochondrial deficits associated with 22qDS or with $\mathrm{SZ}^{5,13,14,33}$, but multiple lines of evidence suggest that these findings are indeed important. First, transgenic mice hemizygous for MRPL4O show deficits in working memory, a SZ-related phenotype, as well as alterations in hippocampal short-term potentiation ${ }^{16}$. Second, alterations in the expression of mitochondrial-functioning genes have been identified in a human post-mortem, laser-capture microdissection study of cortical pyramidal neurons ${ }^{5}$. Interestingly, these include downregulation of MRPL36, MRPL48, and MRPS6, raising the possibility that a mitochondrial translation deficit could be an important aspect of SZ outside of the 22q11DS context ${ }^{5}$. In addition, MRPL18 was highlighted as a gene associated with psychosis based on whole-blood RNA expression in 22q11DS subjects ${ }^{34}$. Third, the capacity that we have demonstrated to study mitochondria defects in iPSC-derived neurons suggests that this system will be amendable to increasingly refined and mechanistic studies on interactions between mitochondria genetics and neuropsychiatric risk, and resilience. 


\section{Acknowledgements}

This work was supported by National Institutes of Health grants R01 MH110185, R01 MH066912, T32 MH19112, and the Brain and Behavior Research Foundation (BBRF).

\section{Author details}

'Department of Psychiatry, Children's Hospital of Philadelphia, Philadelphia, PA, USA. ${ }^{2}$ Department of Psychiatry, The Children's Hospital of Philadelphia and the University of Pennsylvania Perelman School of Medicine, Philadelphia, PA, USA. ${ }^{3}$ Mallinckrodt Pharmaceuticals, Bedminster, NJ, USA. ${ }^{4}$ University of Pennsylvania, Philadelphia, PA, USA. ${ }^{5}$ Department of Psychiatry, Children's Hospital of Philadelphia, Philadelphia, PA, USA. ${ }^{6}$ Department of Psychiatry and Behavioral Sciences, Albert Einstein College of Medicine, Bronx, New York, USA. ${ }^{7}$ Center for Mitochondrial and Epigenomic Medicine, Children's Hospital of Philadelphia and Department of Pediatrics, Division of Human Genetics, Perelman School of Medicine, University of Pennsylvania, Philadelphia, PA, United States. ${ }^{8}$ Department of Pediatrics, The Children's Hospital of Philadelphia and the University of Pennsylvania Perelman School of Medicine, Philadelphia, PA, USA. ${ }^{9}$ Department of Psychiatry, Children's Hospital of Philadelphia and the University of Pennsylvania Perelman School of Medicine, Philadelphia, PA, USA

\section{Conflict of interest}

The authors declare that they have no conflict of interest.

\section{Publisher's note}

Springer Nature remains neutral with regard to jurisdictional claims in published maps and institutional affiliations.

Supplementary Information accompanies this paper at (https://doi.org/ 10.1038/s41398-019-0643-y).

Received: 1 August 2019 Accepted: 11 August 2019 Published online: 18 November 2019

\section{References}

1. Carpenter, W. T. Jr. \& Buchanan, R. W. Schizophrenia. N. Engl. J. Med. 330, 681-690 (1994)

2. Lewis, D. A. Cortical circuit dysfunction and cognitive deficits in schizophreniaimplications for preemptive interventions. Eur. J. Neurosci. 35, 1871-1878 (2012).

3. Whatley, S. A., Curti, D. \& Marchbanks, R. M. Mitochondrial involvement in schizophrenia and other functional psychoses. Neurochem. Res. 21, 995-1004 (1996).

4. Konradi, C. \& Ongur, D. Role of mitochondria and energy metabolism in schizophrenia and psychotic disorders. Schizophr. Res. 187, 1-2 (2017).

5. Arion, D. et al. Transcriptome alterations in prefrontal pyramidal cells distinguish schizophrenia from bipolar and major depressive disorders. Biol. Psychiatry 82, 594-600 (2017).

6. Ni, P. et al. iPSC-derived homogeneous populations of developing schizophrenia cortical interneurons have compromised mitochondrial function. Mol. Psychiatry (2019). https://doi.org/10.1038/s41380-019-0423-3.

7. Brennand, K. et al. Phenotypic differences in hiPSC NPCs derived from patients with schizophrenia. Mol. Psychiatry 20, 361-368 (2015).

8. Scambler, P. J. The 22q11 deletion syndromes. Hum. Mol. Genet. 9, 2421-2426 (2000).

9. Chow, E. W., Watson, M., Young, D. A. \& Bassett, A. S. Neurocognitive profile in 22q11 deletion syndrome and schizophrenia. Schizophr. Res. 87, 270-278 (2006).

10. Sun, D. et al. Large-scale mapping of cortical alterations in 22 q11.2 deletion syndrome: convergence with idiopathic psychosis and effects of deletion size. Mol. Psychiatry (2018). https://doi.org/10.1038/s41380019-0467-4.

11. Maynard, T. M. et al. Mitochondrial localization and function of a subset of 22q11 deletion syndrome candidate genes. Mol. Cell Neurosci. 39, 439-451 (2008).

12. Napoli, E. et al. Mitochondrial citrate transporter-dependent metabolic signature in the 22q11.2 deletion syndrome. J. Biol. Chem. 290, 23240-23253 (2015).

13. Gokhale, A. et al. Systems analysis of the 22 q11.2 microdeletion syndrome converges on a mitochondrial interactome necessary for synapse function and behavior. J. Neurosci. 39, 3561-3581 (2019).

14. Fernandez, A. et al. Mitochondrial dysfunction leads to cortical underconnectivity and cognitive impairment. Neuron 102, 1127-1142.e3 (2019).

15. Carrera, N. et al. Association study of nonsynonymous single nucleotide polymorphisms in schizophrenia. Biol. Psychiatry 71, 169-177 (2012).

16. Devaraju, P. et al. Haploinsufficiency of the 22q11.2 microdeletion gene Mrpl40 disrupts short-term synaptic plasticity and working memory through dysregulation of mitochondrial calcium. Mol. Psychiatry 22, 1313-1326 (2017).

17. Lin, M. et al. Integrative transcriptome network analysis of iPSC-derived neurons from schizophrenia and schizoaffective disorder patients with 22q11.2 deletion. BMC Syst. Biol. 10, 105 (2016).

18. Vorstman, J. A. et al. MLPA: a rapid, reliable, and sensitive method for detection and analysis of abnormalities of 22q. Hum. Mutat. 27, 814-821 (2006).

19. Zhang, Y. et al. Rapid single-step induction of functional neurons from human pluripotent stem cells. Neuron 78, 785-798 (2013).

20. Spinazzi, M., Casarin, A., Pertegato, V., Salviati, L. \& Angelini, C. Assessment of mitochondrial respiratory chain enzymatic activities on tissues and cultured cells. Nat. Protoc. 7, 1235-1246 (2012).

21. Birch-Machin, M. A. \& Turnbull, D. M. Assaying mitochondrial respiratory complex activity in mitochondria isolated from human cells and tissues. Methods Cell Biol. 65, 97-117 (2001).

22. Ran, F. A. et al. Genome engineering using the CRISPR-Cas9 system. Nat Protoc. 8, 2281-2308 (2013).

23. Wallace, D. C. Diseases of the mitochondrial DNA. Annu. Rev. Biochem. 61, 1175-1212 (1992)

24. Boczonadi, V. \& Horvath, R. Mitochondria: impaired mitochondrial translation in human disease. Int. J. Biochem. Cell Biol. 48, 77-84 (2014).

25. Jia, L., Kaur, J. \& Stuart, R. A. Mapping of the Saccharomyces cerevisiae Oxa1mitochondrial ribosome interface and identification of MrpL40, a ribosomal protein in close proximity to Oxa1 and critical for oxidative phosphorylation complex assembly. Eukaryot. Cell 8, 1792-1802 (2009).

26. Avramopoulos, D. Recent advances in the genetics of schizophrenia. Mol. Neuropsychiatry 4, 35-51 (2018).

27. Ursini, G. et al. Convergence of placenta biology and genetic risk for schizophrenia. Nat. Med. 24, 792-801 (2018).

28. Van, L., Boot, E. \& Bassett, A. S. Update on the 22q11.2 deletion syndrome and its relevance to schizophrenia. Curr. Opin. Psychiatry 30, 191-196 (2017).

29. Chatzispyrou, I. A., Held, N. M., Mouchiroud, L., Auwerx, J. \& Houtkooper, R. H. Tetracycline antibiotics impair mitochondrial function and its experimental use confounds research. Cancer Res. 75, 4446-4449 (2015).

30. Gulyas, A. I., Buzsaki, G., Freund, T. F. \& Hirase, H. Populations of hippocampal inhibitory neurons express different levels of cytochrome c. Eur. J. Neurosci. 23, 2581-2594 (2006).

31. Bitanihirwe, B. K. \& Woo, T. U. Oxidative stress in schizophrenia: an integrated approach. Neurosci. Biobehav. Rev. 35, 878-893 (2011).

32. Steullet, $P$. et al. Oxidative stress-driven parvalbumin interneuron impairment as a common mechanism in models of schizophrenia. Mol. Psychiatry 22, 936-943 (2017).

33. Motahari, Z., Moody, S. A., Maynard, T. M. \& LaMantia, A. S. In the line-up: deleted genes associated with DiGeorge/22q11.2 deletion syndrome: are they all suspects? J. Neurodev. Disord. 11, 7 (2019).

34. Jalbrzikowski, M. et al. Transcriptome profiling of peripheral blood in 22 q11.2 deletion syndrome reveals functional pathways related to psychosis and autism spectrum disorder. PLOS ONE 10, e0132542 (2015). 